\title{
PROCESSOS FÍSICO-QUÍMICOS EM DRENAGEM ÁCIDA DE MINA EM MINERAÇÃO DE CARVÃO NO SUL
} DO BRASIL

\author{
Veridiana Polvani Campaner e Wanilson Luiz-Silva* \\ Departamento de Geologia e Recursos Naturais, Instituto de Geociências, Universidade Estadual de Campinas, CP 6152, \\ 13083-970 Campinas - SP, Brasil
}

Recebido em 11/3/08; aceito em 14/8/08; publicado na web em 18/12/08

\begin{abstract}
PHYSICO-CHEMICAL PROCESSES IN ACID MINE DRAINAGE IN COAL MINING, SOUTH BRAZIL. Acid mine drainage generated from coal mine showed a $\mathrm{pH}$ of 3.2, high concentrations of $\mathrm{SO}_{4}^{2-}, \mathrm{Al}, \mathrm{Fe}, \mathrm{Mn}, \mathrm{Zn}$ and minor $\mathrm{As}, \mathrm{Cd}, \mathrm{Co}, \mathrm{Cr}, \mathrm{Cu}, \mathrm{Ni}$ and $\mathrm{Pb}$. The major reduction in the concentration occurred for $\mathrm{Al}, \mathrm{As}, \mathrm{Cr}, \mathrm{Fe}$ and $\mathrm{Pb}$ after the treatment with $\mathrm{CaO}$. The evolution of these acid waters within the tributary stream showed decreasing concentration for all soluble constituents, except Al. This natural attenuation was controlled by $\mathrm{pH}$ (6.4 to 10.8) as a result of concurrent mixing with tributary stream and reaction with local bedrock that contains limestone. Aluminum increasing concentration during this evolution seems to be related to an input of Al-enriched waters due to the leaching of silicate minerals in alkaline conditions.
\end{abstract}

Keywords: acid mine drainage; metal contamination; treatment with $\mathrm{CaO}$.

\section{INTRODUÇÃO}

Drenagem ácida de mina (DAM) é um grave problema oriundo das atividades de mineração, tanto de metais quanto de carvão, por causar a degradação da qualidade de águas superficiais e subterrâneas, solos e sedimentos. A DAM é gerada especialmente pela oxidação de minerais de sulfeto e tem alta capacidade de lixiviação de elementos presentes no minério e nas rochas circundantes à área minerada. Estas drenagens são caracterizadas por $\mathrm{pH}$ baixo, alta condutividade e altas concentrações de $\mathrm{Al}, \mathrm{Fe}, \mathrm{Mn}$, entre outros metais e metalóides, que auxiliam na acidez destas drenagens, uma vez que podem sofrer reações de hidrólise. ${ }^{1}$ Os minerais presentes nas rochas explotadas, o tipo e a quantidade do sulfeto oxidado condicionam os níveis de contaminação por metais e metalóides associados com a DAM, e são específicos de cada ambiente. ${ }^{1,2}$ Dentre os sulfetos freqüentemente encontrados e que podem produzir drenagem ácida estão a pirita $\left(\mathrm{FeS}_{2}\right)$, arsenopirita $(\mathrm{FeAsS})$, calcopirita $\left(\mathrm{CuFeS}_{2}\right)$ e calcocita $\left(\mathrm{Cu}_{2} \mathrm{~S}\right)$, sendo a primeira a mais comum. ${ }^{3}$

O reconhecimento dos riscos ambientais da DAM tem conduzido ao desenvolvimento de várias técnicas para minimizar o impacto destas correntes ácidas nos ecossistemas. Os sistemas convencionais de neutralização de DAM que utilizam reagentes alcalinos (e.g. CaO e $\mathrm{CaCO}_{3}$ ) são os mais empregados em áreas de mineração. Estes sistemas consistem no aumento do $\mathrm{pH}$ e na remoção de metais na forma de hidróxidos. A principal característica da neutralização com reagentes alcalinos é a formação de resíduos (lama) decorrentes do processo de precipitação dos sólidos. Em geral, o processo de precipitação de constituintes químicos é em função do $\mathrm{pH}$. Entretanto, outros fatores podem contribuir neste processo, como força iônica, temperatura, Eh, estado de oxidação dos metais, concentrações dos metais em solução e suas interações com sólidos precipitados. ${ }^{4}$

Estudos prévios apontaram que a eficiência da neutralização por reagentes carbonáticos é fortemente influenciada por elevadas concentrações de ferro em solução. ${ }^{5,6} \mathrm{O}$ revestimento das partículas dos reagentes por precipitados de hidróxidos de Fe pode afetar a taxa de reações químicas que consomem $\mathrm{H}^{+}$da solução. ${ }^{7} \mathrm{O}$ estado de oxidação

\footnotetext{
*e-mail: wanilson@ige.unicamp.br
}

do ferro e sua remoção da DAM dependem muito do controle do pH no sistema de neutralização. Em drenagens ácidas, onde o valor de $\mathrm{pH}$ é baixo, predomina a forma iônica $\mathrm{Fe}^{2+}$ sobre a $\mathrm{Fe}^{3+}$. O ferro férrico é facilmente removido da drenagem ácida devido à sua baixa solubilidade e precipitação incipiente como hidróxido em $\mathrm{pH} 3 .{ }^{5} \mathrm{O}$ íon $\mathrm{Fe}^{2+}$ também pode ser removido da solução como hidróxido, embora em valores de $\mathrm{pH}<7,5$ a precipitação seja lenta e incompleta. ${ }^{8}$ Devido à baixa solubilidade dos reagentes carbonáticos em $\mathrm{pH}>7$, condições físico-químicas para a pré-oxidação do $\mathrm{Fe}^{2+}$ para $\mathrm{Fe}^{3+}$ são necessárias para a remoção efetiva do ferro da drenagem ácida. ${ }^{9}$

A remoção de manganês da DAM por sistemas convencionais de neutralização, por outro lado, tem apresentado baixa eficiência em diversos estudos. ${ }^{6,10}$ Em condições oxidantes, o $\mathrm{Mn}^{2+}$ deveria sofrer oxidação espontânea para manganês trivalente ou tetravalente e em seguida precipitar na forma de óxidos. Porém, isto requer elevada quantidade de energia, tornando lento o processo de oxidação deste metal, especialmente em $\mathrm{pH}<8 .{ }^{11,12} \mathrm{Em} \mathrm{pH}$ próximo a 7, essa oxidação pode ser catalisada por meio de microrganismos, o que não procede na presença de concentrações elevadas de $\mathrm{Fe}^{2+}$ na solução. ${ }^{10,11} \mathrm{De}$ modo geral, o íon ferroso reduz a capacidade de oxidação do $\mathrm{Mn}^{2+}$ e a remoção significativa de Mn na drenagem ácida ocorrerá apenas quando as condições forem favoráveis para baixar a concentração de $\mathrm{Fe}^{2+}$.

No Brasil, poucos estudos foram realizados sobre drenagens ácidas e têm sido focados especialmente em regiões carboníferas no sul do país. Pampêo et al., ${ }^{13}$ por exemplo, encontraram baixos valores de $\mathrm{pH}$ e elevados níveis de condutividade, Eh, sólidos dissolvidos, $\mathrm{Al}, \mathrm{Ca}, \mathrm{Fe}, \mathrm{Mg}, \mathrm{Mn}$ e $\mathrm{SO}_{4}{ }^{2-}$ em águas fluviais da Bacia Hidrográfica Fiorita (Siderópolis - SC). Esta situação foi atribuída à exposição atmosférica de rejeitos de mineração de carvão. Soares et $a l .{ }^{14}$ discutiram a possibilidade de utilização de cinzas da combustão do carvão, em combinação com carbonato de cálcio $\left(\mathrm{CaCO}_{3}\right)$, para correção da drenagem ácida decorrente da oxidação da pirita em estéreis da mineração em Candiota (RS). Na região de Figueira (PR), Flues et al. ${ }^{15}$ discutiram a presença de chuvas ácidas em decorrência das atividades carboníferas na região, enquanto Fungaro e Izidoro ${ }^{16}$ estudaram a descontaminação de drenagem ácida pelo processo de troca iônica. Estes últimos utilizaram zeólita sintetizada a partir de 
cinzas de carvão da própria região. Em adição, Shuqair ${ }^{17}$ estudou a contaminação do solo e da água subterrânea por elementos tóxicos de rejeitos da mineração de carvão de Figueira. Em geral, os trabalhos realizados no entorno de minerações carboníferas do Brasil têm estudado pouco os processos de tratamento de DAM e as conseqüências físico-químicas ao ambiente.

Os objetivos do presente trabalho foram investigar processos físico-químicos de precipitação de metais e arsênio em sistema de tratamento de drenagem ácida de mina de carvão com $\mathrm{CaO}$, e averiguar a evolução das águas ácidas em drenagem natural em área com presença de rochas calcárias.

\section{PARTE EXPERIMENTAL}

\section{Área de estudo e o sistema de neutralização da DAM}

A área de estudo localiza-se no município de Figueira, nordeste do Estado do Paraná (Figura 1), no domínio da Bacia Sedimentar do Paraná. Dentre as unidades geológicas desta bacia que ocorrem na área, a Formação Rio Bonito destaca-se por conter camadas de carvão. Segundo Schneider et al. ${ }^{18}$ a estratigrafia desta unidade constitui-se de uma porção basal arenosa; uma intermediária, que compreende siltitos e folhelhos intercalados com camadas de arenitos e leitos de rochas calcárias; e uma camada superior, constituída por arenitos intercalados com leitos de argila e folhelhos carbonosos, onde estão localizados leitos de carvão que podem ser explotados economicamente.

As jazidas de carvão da região têm sido explotadas desde a década de 40, as quais proporcionaram a partir de 1959 a instalação de uma usina termelétrica na área de estudo. A explotação do minério ocorreu tanto a céu aberto quanto de forma subterrânea. Atualmente, há apenas uma mina de carvão em atividade (subterrânea), mas várias outras desativadas somam dezenas de quilômetros de túneis e escavações de superfície, todos preenchidos por águas pluviais e subterrâneas. A única mina atualmente em atividade foi acessada por um plano inclinado denominado 07, com início do processo de lavra em 2001. O carvão da mina 07 , como ficou conhecida, é caracterizado como betuminoso, alto volátil $\mathrm{A}$, com baixo teor de água $(<3 \%)$, coloração negra brilhante e com elevado poder calorífico. O carvão é extraído pelo método de câmaras e pilares, com dimensões de $5 \times 5 \mathrm{~m}$. A frente de produção é composta por aproximadamente 9 galerias com altura variando de 1 a 1,8 m e largura de $5 \mathrm{~m}$. Os leitos de carvão estão dispostos horizontalmente em três camadas intercaladas com camadas de siltito. Cada camada não ultrapassa $0,50 \mathrm{~m}$, mas o minério (carvão e siltito) pode alcançar 1,42 m de altura. Destas camadas são produzidas aproximadamente $97 \mathrm{mil} \mathrm{t} \mathrm{ano}^{-1}$ de carvão. ${ }^{19}$

Dados de sondagem na área da mina 07 (Carbonífera do Cambuí, inédito) revelaram sobre as camadas do minério um pacote de rochas siliciclásticas e químicas (38 m, aproximadamente), formado por alternâncias de camadas métricas de arenitos (raramente piritosos) e siltitos (raramente carbonosos), ambas ocasionalmente calcíferas na base. Nesta seqüência ocorrem leitos centimétricos de calcários impuros. Esse pacote sedimentar é responsável pelo aporte de água subterrânea que flui continuamente para as galerias da mina (água ácida). Um sistema de bombeamento drena a água para a superfície, onde se inicia o tratamento com $\mathrm{CaO}$ industrial (agente neutralizante). Em seguida, a água é conduzida por canaletas seladas para dois tanques interligados e impermeabilizados com argila, com capacidade de $2268 \mathrm{~m}^{3}$. A água reside em cada tanque por um tempo de aproximadamente $24 \mathrm{~h}$ e ao final desse processo, a água é drenada para o córrego da Mina, tributário do rio Laranjinha. O rio Laranjinha é o principal da região de Figueira e é a fonte de abastecimento de água do município. O material precipitado nos tanques (lama) é transportado e depositado em galerias desativadas no interior da mina 07.

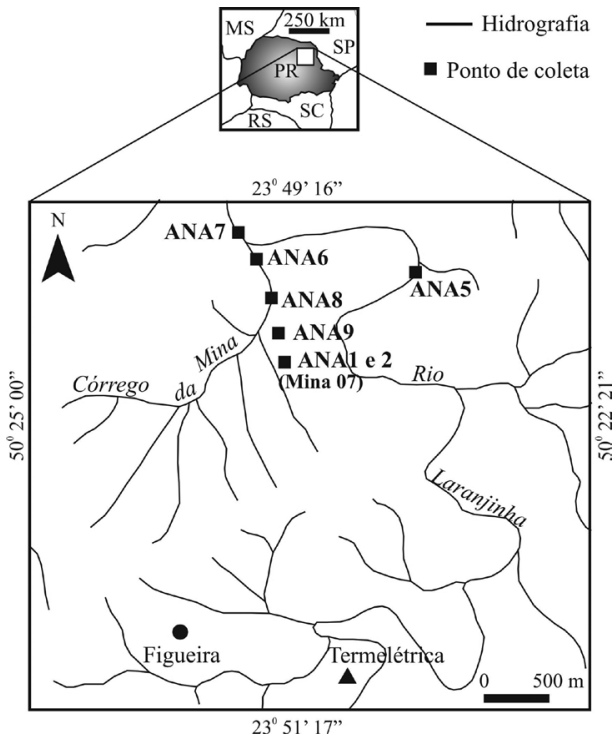

Figura 1. Localização da área de estudo e pontos de amostragem. ANA1 DAM do interior da mina de carvão; ANA2 - DAM após o tratamento com CaO; ANA8 e ANA6 são amostras do córrego da Mina a 200 e 700 m, respectivamente, do ponto de descarga da DAM; ANA5 e ANA7 são amostras do rio Laranjinha, antes e após, respectivamente, o ponto de confluência com o córrego da Mina; ANA9 - água subterrânea, poço a jusante da mina 07

\section{Amostragem e preparação das amostras}

As amostras de água foram coletadas em setembro de 2006 (período hídrico de baixo fluxo), utilizando garrafas PET (garrafas comerciais de água mineral gaseificada ${ }^{20}$ esvaziadas no local da coleta e lavadas com uma fração da água a ser amostrada. Sete pontos de coleta de amostras foram considerados neste estudo (Figura 1). Durante a amostragem, parâmetros físico-químicos foram medidos com os equipamentos, previamente calibrados, Horiba U10 (sólidos totais dissolvidos e temperatura), Digimed DM-3 (pH e Eh) e Digimed DM-2 (condutividade). As medidas de redox foram realizadas com eletrodo combinado de platina e as de $\mathrm{pH}$, com eletrodo combinado de vidro. Em ambos os casos, o eletrodo de referência foi $\mathrm{Ag}: \mathrm{AgCl}([\mathrm{KCl}]=3 \mathrm{~mol} / \mathrm{L})$. Os valores de Eh medidos foram recalculados para o potencial padrão do hidrogênio (Standard Hydrogen Electrode - SHE), utilizando a seguinte equação:

$\mathrm{Eh}_{\text {SHE }}=\mathrm{Eh}_{\text {medido }}+209-0,7\left(\mathrm{t}-25^{\circ} \mathrm{C}\right) \mathrm{mV}$

onde, $209 \mathrm{mV}$ é o potencial do eletrodo de referência, relativo ao $S H E$, e 0,7 é um fator de correção, função da temperatura de aquisição do $E .^{21}$

Após a coleta, a água foi filtrada em membrana de acetato de celulose Millipore ${ }^{\circledR}$, com poros de $0,22 \mu \mathrm{m}$, para posteriores análises de cátions e ânions. Apenas as amostras destinadas à análise de cátions foram imediatamente estabilizadas, utilizando $\mathrm{HNO}_{3}$ Merck ${ }^{\circledR}$ suprapuro $(1 \% \mathrm{v} / \mathrm{v}) .{ }^{22}$ Todas as amostras foram mantidas sob refrigeração a $4{ }^{\circ} \mathrm{C}$, até as análises em laboratório.

Amostras de lama dos dois tanques de decantação do processo de neutralização da DAM foram coletadas $(\sim 3,0 \mathrm{~kg})$, assim como 4 amostras de carvão da mina 07. Para fins analíticos, as amostras da lama foram homogeneizadas e secadas em estufa a $40{ }^{\circ} \mathrm{C}$ e as de carvão, pulverizadas em potes e bolas de ágata. Testes de peneiramento revelaram que a lama era composta integralmente por partículas $<63 \mu \mathrm{m}$. 


\section{Análises químicas}

A alcalinidade, representada pelo ânion $\mathrm{HCO}_{3}{ }^{-}$, foi medida em amostras de água de drenagens naturais (ANA8, ANA6, ANA7 e ANA5) in situ por titulação de $100 \mathrm{~mL}$ da amostra, usando como indicador azul de bromocresol e $\mathrm{H}_{2} \mathrm{SO}_{4}(0,16 \mathrm{~N})$ como reagente. Análises químicas multi-elementares (Al, $\mathrm{As}, \mathrm{Ca}, \mathrm{Cd}, \mathrm{Co}, \mathrm{Cr}, \mathrm{Cu}$, $\mathrm{Fe}, \mathrm{Mg}, \mathrm{Mn}, \mathrm{Ni}, \mathrm{Pb}, \mathrm{Sr}$ e $\mathrm{Zn}$ ) foram realizadas em todas as amostras de água, lama de decantação e de carvão. No caso do carvão, a análise de enxofre total foi incluída, enquanto na água o $\mathrm{SO}_{4}{ }^{2-}$ foi quantificado. As medidas nas amostras de água foram realizadas via ICPMS (exceto o $\mathrm{SO}_{4}^{2-}$, por cromatografia iônica), no Laboratório de Geoquímica do IG, Unicamp. Nas amostras da lama de decantação e carvão, análises químicas foram realizadas via ICPMS, no Acme Analytical Laboratories, Vancouver/Canadá, após digestão a quente de 0,25 g do material em solução multi-ácida $\left(2: 2: 1: 1 \mathrm{H}_{2} \mathrm{O}\right.$ $\mathrm{HF}-\mathrm{HClO}_{4}-\mathrm{HNO}_{3}$ ). Para o controle da qualidade analítica, materiais de referência (SRM1640-NIST e LKSD3-CANMET nas amostras de água e sedimento, respectivamente - Tabela 1), duplicatas de amostras (diferenças percentuais relativas inferiores a 15\%) e brancos foram empregados.

\section{Tratamento dos dados}

As concentrações de $\mathrm{CO}_{3}{ }^{2-}$ nas amostras de drenagem natural (ANA8, ANA6, ANA7 e ANA5) foram estimadas por meio do programa GwCHART, ${ }^{23}$ utilizando dados de $\mathrm{HCO}_{3}^{-}, \mathrm{pH}$ e temperatura obtidos previamente. A modelagem geoquímica foi elaborada pelo programa PHREEQC Interactive $2.6,{ }^{24}$ a partir das condições físicoquímicas das amostras de água, permitindo calcular as espécies aquosas. Para o programa de modelagem geoquímica, os potenciais de redox foram recalculados para valores de $\mathrm{p} \varepsilon$, os quais indicam a atividade do elétron (-log $[\bar{e}])$ no equilíbrio químico. ${ }^{25}$ A conversão do Eh em pe foi obtida pela equação:

$\mathrm{p} \varepsilon=\mathrm{Eh}_{\text {SHE }}(\mathrm{F} / 2,303 \mathrm{RT})$ onde F é a constante de Faraday ( $\left.96485 \mathrm{C} \mathrm{mol}^{-1}\right)$, R é a constante dos gases $\left(8,315 \mathrm{~J} \mathrm{~K} \mathrm{~mol}^{-1}\right)$ e T é a temperatura em Kelvin. ${ }^{26}$ Para a temperatura de $25^{\circ} \mathrm{C}$, a expressão reduz-se a p $\varepsilon=16,91 \mathrm{Eh}_{S H E}$.

Para cada elemento químico do carvão foi calculado o fator de concentração (FC), o qual é a razão entre a concentração do elemento de interesse na amostra (carvão) e sua concentração média na crosta continental (valor de Clarke), ${ }^{27}$ procedimento bastante utilizado na literatura. $^{28,29}$

\section{RESULTADOS E DISCUSSÃO}

\section{Geoquímica do carvão}

A Tabela 2 apresenta as concentrações de 15 constituintes químicos do carvão da área de estudo e os seus fatores de concentração (FC), os valores de Clarke e as concentrações químicas em carvões do sul do Brasil. Em comparação com a média crustal (Clarke), as concentrações de As do carvão da área de estudo foram muito elevadas (253 a $2176 \mathrm{mg} \mathrm{kg}^{-1}$ ), com FC acima de 100. Para o $\mathrm{Cd}$ e $\mathrm{Pb}$ os valores de FC variaram entre 3,1-35,5 e 14,7-42,5, respectivamente. Valores de FC levemente acima da unidade também foram observados para o Fe (2,5-15,9) e Zn $(0,7-6,8)$. Para os demais elementos investigados, os valores de $\mathrm{FC}$ foram $<1$. As concentrações de $\mathrm{As}$ e $\mathrm{Pb}$ no carvão de Figueira foram muito superiores aos carvões de Criciúma (SC) e Candiota (RS) (Tabela 2). Em adição, as elevadas concentrações de As nas amostras de carvão de Figueira são notórias e comparáveis com outros estudos, como por exemplo, na Grã Bretanha (1254 mg kg$\left.{ }^{-1}\right),{ }^{30}$ no Canadá $\left(>1400 \mathrm{mg} \mathrm{kg}^{-1}\right)^{31}$ e na China $\left(2226 \mathrm{mg} \mathrm{kg}^{-1}\right){ }^{32}$

\section{Geoquímica de drenagem ácida neutralizada com $\mathrm{CaO}$}

A Tabela 3 mostra os parâmetros físico-químicos e as concentrações de constituintes químicos das amostras de água da área de estudo. A amostra ANA1 (interior da mina 07) apresentou pH 3,2, Eh $625 \mathrm{mV}$, elevada condutividade $(4850 \mu \mathrm{S} / \mathrm{cm})$, e concentrações de

Tabela 1. Concentrações de elementos químicos em materiais de referência para água (SRM1640, valores em $\mu g \mathrm{~L}^{-1}$ ) e sedimento (LKSD3, valores em $\mathrm{mg} \mathrm{kg}^{-1}$ )

\begin{tabular}{lcccccc}
\hline & \multicolumn{3}{c}{ SRM1640-NIST } & \multicolumn{3}{c}{ LKSD3-CANMET } \\
& Valor de Referência & Valor Obtido & Recuperação (\%) & Valor de Referência & Valor Obtido & Recuperação (\%) \\
\hline $\mathrm{Al}$ & 52 & 55,9 & 107,5 & 66110 & 65300 & 98,8 \\
$\mathrm{Ca}$ & 7045 & 7431 & 105,5 & 16400 & 17700 & 107,9 \\
$\mathrm{Fe}$ & 34,3 & 35,1 & 102,2 & 40000 & 42000 & 105 \\
$\mathrm{Mg}$ & 5819 & 5788 & 99,5 & 1206 & 1130 & 93,7 \\
$\mathrm{~S}$ & - & - & - & 1400 & 1600 & 114,3 \\
$\mathrm{As}$ & 26,7 & 29 & 108,6 & 27 & 23,4 & 86,7 \\
$\mathrm{Cd}$ & 22,8 & 24,5 & 107,5 & 5,6 & 5,7 & 101,8 \\
$\mathrm{Co}$ & 20,3 & 22,8 & 112,4 & 30 & 32,8 & 109,3 \\
$\mathrm{Cr}$ & 38,6 & 41,9 & 108,4 & 87 & 76 & 87,4 \\
$\mathrm{Cu}$ & 85,2 & 97,2 & 114,1 & 35 & 38,2 & 109 \\
$\mathrm{Mn}$ & 122 & 134 & 110 & 1440 & 1469 & 102 \\
$\mathrm{Ni}$ & 27,4 & 26,2 & 95,6 & 47 & 50,8 & 108,1 \\
$\mathrm{~Pb}$ & 27,9 & 30,3 & 108,6 & 29 & 32,8 & 113,1 \\
$\mathrm{Sr}$ & 124 & 132 & 105,9 & 240 & 270 & 112,5 \\
$\mathrm{Zn}$ & 53,2 & 53,2 & 100 & 152 & 166,1 & 109,3 \\
\hline
\end{tabular}


Tabela 2. Concentrações de alguns elementos ( $\mathrm{mg} \mathrm{kg}^{-1}$ quando não especificada) nas amostras de carvão da área de estudo e seus fatores de concentração (FC). Valores de Clarke (média crustal) e concentrações em carvões de Criciúma (SC) e Candiota (RS)

\begin{tabular}{|c|c|c|c|c|c|c|c|c|}
\hline & Carv-1 & Carv-2 & Carv-3 & Carv-4 & $\mathrm{FC}$ & Clarke $^{\mathrm{a}}$ & Criciúmab $^{\mathrm{b}}$ & Candiota $^{\mathrm{c}}$ \\
\hline $\mathrm{A} 1 \%$ & 3,13 & 1,47 & 1,98 & 1,05 & $0,1-0,3$ & 8,4 & - & - \\
\hline $\mathrm{Ca} \%$ & 2,1 & 0,06 & 0,17 & 0,42 & $0,01-0,4$ & 5,3 & - & - \\
\hline $\mathrm{Fe} \%$ & 3,75 & 11,2 & 1,98 & 1,77 & $2,5-15,9$ & 0,71 & - & - \\
\hline $\mathrm{Mg} \%$ & 0,19 & 0,02 & 0,11 & 0,03 & $0,01-0,06$ & 3,2 & - & - \\
\hline $\mathrm{S} \%$ & 4,45 & $>10$ & 2,96 & 3,11 & - & - & - & - \\
\hline As & 381 & 2176 & 253 & 279 & $253-2176$ & 1 & 35,1 & 9,85 \\
\hline $\mathrm{Cd}$ & 0,3 & 0,75 & 3,48 & 0,69 & $3,1-35,5$ & 0,09 & 2,6 & 0,1 \\
\hline Co & 7,7 & 22,5 & 8,5 & 4,8 & $0,2-0,8$ & 29 & 10,3 & 9,26 \\
\hline $\mathrm{Cr}$ & 47 & 16 & 28 & 21 & $0,09-0,2$ & 185 & 73 & 35,5 \\
\hline $\mathrm{Cu}$ & 26,1 & 16,1 & 30,8 & 22,1 & $0,2-0,4$ & 75 & 33,4 & 12,3 \\
\hline $\mathrm{Mn}$ & 392 & 87 & 91 & 114 & $0,06-0,3$ & 1400 & - & 214 \\
\hline $\mathrm{Ni}$ & 15,8 & 24,6 & 24,9 & 20,6 & $0,1-0,2$ & 105 & 24,1 & 15,5 \\
\hline $\mathrm{Pb}$ & 135 & 340 & 119 & 118 & $14,7-42,5$ & 8 & 49,6 & 14 \\
\hline $\mathrm{Sr}$ & 100 & 106 & 51 & 15 & $0,06-0,4$ & 260 & 56,3 & 78,7 \\
\hline $\mathrm{Zn}$ & 54,4 & 97,4 & 544 & 175 & $0,7-6,8$ & 80 & 474 & 75,4 \\
\hline
\end{tabular}

${ }^{\mathrm{a}}$ ref. $27,{ }^{\mathrm{b}}$ ref. 33 , ref. 34

$83 \mathrm{mg} \mathrm{L}^{-1} \mathrm{Al}, 473 \mathrm{mg} \mathrm{L}^{-1} \mathrm{Fe}, 11,8 \mathrm{mg} \mathrm{L}^{-1} \mathrm{Mn}$ e $2969 \mathrm{mg} \mathrm{L}^{-1} \mathrm{SO}_{4}{ }^{2-}$, características típicas de DAM. ${ }^{35}$

O incremento substancial do $\mathrm{pH}$ (de 3,2 em ANA1 para 4,6 em ANA2) e decréscimo no Eh (de 625 para $445 \mathrm{mV}$ ) na DAM póstratamento com $\mathrm{CaO}$ condicionaram a diminuição nas concentrações de todos os elementos da água, exceto $\mathrm{Ca}, \mathrm{Mg}$ e $\mathrm{Sr}$ que aumentaram (Tabela 3). O incremento destes elementos em ANA2 deve ser contribuição do agente neutralizante $(\mathrm{CaO})$, um produto industrializado a partir de rochas calcárias da Bacia do Paraná. No momento em que a DAM (pH original de 3,2 ) recebeu a dosagem de $\mathrm{CaO}$ (início do processo de tratamento), o $\mathrm{pH}$ foi subitamente elevado a 11,1 (não mostrado na Tabela 3), o qual diminuiu significativamente para 4,6 (amostra ANA2) após o tempo de residência do efluente nos dois tanques de decantação ( $24 \mathrm{~h}$ em cada tanque). Após esse processo, embora a carga de componentes químicos tenha diminuído, as concentrações de alguns constituintes continuaram elevadas, como sugerem os níveis de $\mathrm{Al}\left(15,5 \mathrm{mg} \mathrm{L}^{-1}\right), \mathrm{Fe}$ (256 mg L L $)$, Mn (10,9 mg $\left.\mathrm{L}^{-1}\right)$ e $\mathrm{Zn}\left(10,9 \mathrm{mg} \mathrm{L}^{-1}\right)$ na amostra ANA2. A insuficiência de $\mathrm{CaO}$ no processo de neutralização da DAM pode ter contribuído para isso, como indica o elevado teor de $\mathrm{SO}_{4}^{2-}\left(2592 \mathrm{mg} \mathrm{L}^{-1}\right)$ nesta amostra. É importante salientar que as concentrações deste íon são minimizadas pelo sistema de neutralização apenas quando a solubilidade de $\mathrm{CaSO}_{4}$ é excedida pela adição de cálcio, ${ }^{1}$ o que parece não ter ocorrido de forma significativa neste caso.

A Figura 2 apresenta a relação de alguns constituintes na DAM antes (ANA1) e após o tratamento com CaO (ANA2). Nota-se nesta figura uma forte relação entre a remoção de elementos e o pH de hidrólise de cada espécie química (considerou-se o principal estado de valência), o qual representa um valor específico de $\mathrm{pH}$, acima do qual o elemento não é mais encontrado na forma solúvel..$^{36}$ Elementos como Cd, Co, Mn, Ni e Zn (pH de hidrólise entre 6,7 e 8,8) mostraram baixa remoção no processo de neutralização, ao passo que $\mathrm{Al}, \mathrm{As}, \mathrm{Cr}$, $\mathrm{Cu}, \mathrm{Fe}$ e $\mathrm{Pb}$ (pH de hidrólise entre 4,1 e 6, exceto As não fornecido) apresentaram expressiva taxa de remoção. O comportamento destes elementos sugere taxa de precipitação inversamente proporcional ao $\mathrm{pH}$ de hidrólise, embora outros fatores pudessem influenciar na remoção de metais e metalóides em solução. No caso do As (pH de hidrólise não documentado na literatura), os resultados de vários estudos ${ }^{37-39}$ sobre a remoção deste metalóide de drenagens ácidas sugerem forte tendência de retenção do mesmo em precipitados de oxi-hidróxidos de Fe, que acabam influenciando na sua mobilidade.

A Tabela 4 apresenta as concentrações totais de alguns elementos químicos na lama de decantação dos tanques do sistema de tratamento com $\mathrm{CaO}$. Para alguns elementos ( $\mathrm{Cd}, \mathrm{Co}, \mathrm{Mn}, \mathrm{Ni}$ e $\mathrm{Zn})$, as concentrações são maiores na lama de decantação do segundo tanque, em comparação com o primeiro tanque. Isto pode ser conseqüência da cinética de remoção destes constituintes da solução (maior pH de hidrólise, menor taxa de precipitação), como mostra a Figura 2. Por outro lado, elementos com maior facilidade de remoção da solução (Al, As, Cr, Cu, Fe e $\mathrm{Pb}$; Figura 2) não apresentaram diferenças significativas nas concentrações da lama dos dois tanques, sugerindo continuidade no processo de precipitação no decorrer do sistema de neutralização.

A entrada do efluente ácido (amostra ANA2, pH 4,6) na rede de drenagem local (córrego da Mina) resultou em um novo sistema

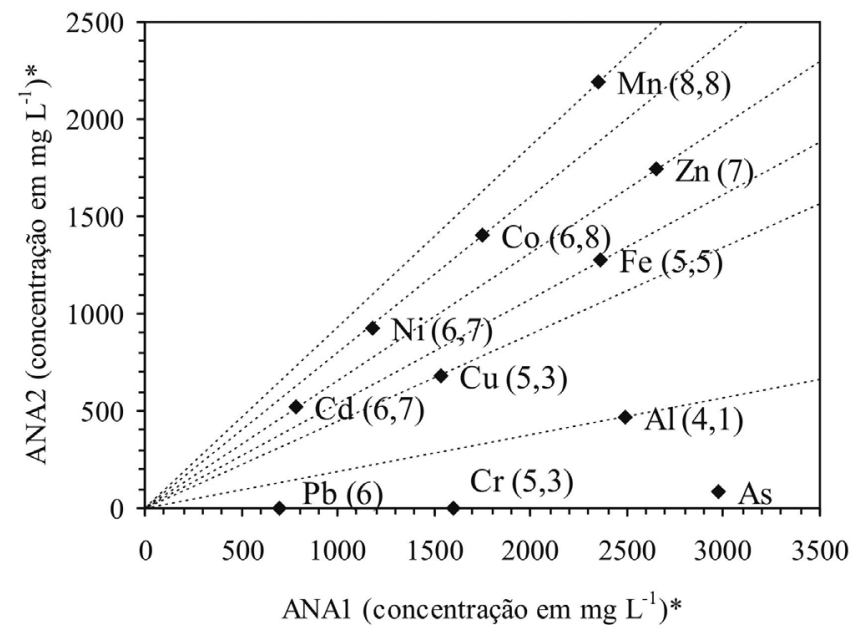

Figura 2. Concentrações de alguns elementos nas amostras ANA1 (DAM antes do tratamento) e ANA2 (DAM pós o tratamento). Entre parênteses consta o pH de hidrólise para cada elemento, ${ }^{40}$ exceto As. *Al (30 vezes a concentração em mg L ${ }^{-1}$ ); As (9000); Cd (13000); Co (3500); Cr (20000); Cu (17000); $\mathrm{Fe}$ (5); $\mathrm{Mn}$ (200); $\mathrm{Ni}$ (950); $\mathrm{Pb}$ (70000); $\mathrm{Zn}(160)$ 
Tabela 3. Parâmetros físico-químicos e constituintes químicos das amostras de água ( $\mathrm{mg} \mathrm{L}^{-1}$, quando não especificado) da área de estudo. Alguns valores $(<)$ estão abaixo do limite mínimo de detecção do instrumento analítico

\begin{tabular}{|c|c|c|c|c|c|c|c|}
\hline & ANA1 & ANA2 & ANA8 & ANA6 & ANA7 & ANA5 & ANA9 \\
\hline$\overline{\mathrm{pH}}$ & 3,2 & 4,6 & 6,4 & 10,8 & 7,3 & 6,2 & 4,1 \\
\hline Eh $(\mathrm{mV})$ & 625 & 445 & 316 & 296 & 394 & 412 & 580 \\
\hline Cond. $(\mu \mathrm{S} / \mathrm{cm})$ & 4850 & 4130 & 2740 & 1770 & 210 & 180 & 1670 \\
\hline TDS & - & - & 2951 & 1736 & 209 & 153 & 1787 \\
\hline $\mathrm{CO}_{3}{ }^{2-*}$ & - & - & 0,004 & 128 & 0,023 & 0,0018 & - \\
\hline $\mathrm{HCO}_{3}^{-}$ & - & - & 39 & 48,8 & 29,3 & 27,3 & - \\
\hline $\mathrm{SO}_{4}^{2-}$ & 2969 & 2592 & 1587 & 773 & 12,6 & 14,4 & 229 \\
\hline $\mathrm{Al}$ & 83 & 15,5 & 0,07 & 0,63 & 0,16 & 0,05 & 4,69 \\
\hline As & 0,33 & 0,01 & $<0,00006$ & $<0,00006$ & 0,001 & 0,001 & 0,004 \\
\hline $\mathrm{Ca}$ & 368 & 682 & 498 & 285 & 8,56 & 5,18 & 54,5 \\
\hline $\mathrm{Cd}$ & 0,06 & 0,04 & 0,01 & $<0,00001$ & $<0,00001$ & $<0,00001$ & 0,002 \\
\hline $\mathrm{Co}$ & 0,5 & 0,4 & 0,07 & $<0,0001$ & $<0,0001$ & $<0,0001$ & 0,02 \\
\hline $\mathrm{Cr}$ & 0,08 & $<0,0001$ & $<0,0001$ & $<0,0001$ & $<0,0001$ & $<0,0001$ & $<0,0001$ \\
\hline $\mathrm{Cu}$ & 0,09 & 0,04 & 0,003 & 0,001 & 0,002 & 0,002 & 0,001 \\
\hline $\mathrm{Fe}$ & 473 & 256 & 17,8 & $<0,003$ & 0,27 & 0,13 & 0,24 \\
\hline $\mathrm{Mg}$ & 133 & 167 & 44,3 & 1,46 & 1,65 & 1,67 & 9,09 \\
\hline $\mathrm{Mn}$ & 11,8 & 10,9 & 2,48 & $<0,001$ & 0,05 & 0,07 & 0,46 \\
\hline $\mathrm{Ni}$ & 1,24 & 0,97 & 0,16 & 0,002 & 0,001 & 0,001 & 0,03 \\
\hline $\mathrm{Pb}$ & 0,01 & $<0,00001$ & $<0,00001$ & $<0,00001$ & 0,0001 & 0,0004 & 0,007 \\
\hline $\mathrm{Sr}$ & 2,9 & 3,49 & 1,72 & 0,86 & 0,04 & 0,03 & 0,26 \\
\hline $\mathrm{Zn}$ & 16,6 & 10,9 & 1,53 & $<0,00002$ & 0,01 & 0,01 & 0,47 \\
\hline
\end{tabular}

*Calculado com o programa GwCHART. ${ }^{23}$

físico-químico, inicialmente com pH 6,4 (amostra ANA8) e menores níveis (relativos à ANA2) de Eh e dos constituintes químicos analisados (Tabela 3). Esta elevação do $\mathrm{pH}$ e decréscimo nas concentrações químicas na amostra ANA8 (200 m a jusante do ponto de descarga da DAM) devem ser resultantes de diluição da DAM na drenagem natural, esta última com pH original de 6,7 (medido a $200 \mathrm{~m}$ a montante da descarga da DAM).

No córrego da Mina, $700 \mathrm{~m}$ a jusante do ponto de descarga do efluente ácido, ocorreu um aumento significativo do $\mathrm{pH}$ (10,8; amostra ANA6). Neste ponto, comparados à amostra ANA8, os níveis de metais dissolvidos na água são ainda menores, exceto Al. O incremento na concentração de $\mathrm{Al}$ (de 0,07 para $0,63 \mathrm{mg} \mathrm{L}^{-1}$ ), neste caso, é atribuído à lixiviação de minerais silicáticos sob condições alcalinas, as quais favoreceram a solubilização do metal. $\mathrm{O}$ aumento do $\mathrm{pH}$ deve ser conseqüência da presença de níveis de calcário em subsuperfície ( $10 \mathrm{~m}$ de profundidade), de acordo com dados de sondagem geológica na mina 07. Assim, águas subterrâneas carbonatadas devem alimentar o córrego da Mina, especialmente a jusante do ponto ANA8. A amostra de água subterrânea coletada em um poço a montante deste ponto (amostra ANA9; Tabela 3) apresentou pH ácido $(4,1)$, condições oxidantes e elevadas condutividade e concentração de $\mathrm{SO}_{4}{ }^{2-}$, provavelmente decorrentes da oxidação de minerais sulfetados. ${ }^{41}$ Entretanto, baixas concentrações de metais e As foram encontradas nesta amostra.

\section{O sistema carbonático}

Os processos geoquímicos que influenciaram o comportamento das concentrações dos elementos no córrego da Mina podem ser entendidos como um complexo sistema que envolveu o $\mathrm{CaO}$ lançado durante o tratamento da DAM e as camadas de calcário locais.
Tabela 4. Concentração total de alguns elementos químicos ( $\mathrm{mg} \mathrm{kg}^{-1}$, quando não especificada) na lama dos tanques de decantação 1 e 2 (amostras S2A e S2B, respectivamente)

\begin{tabular}{lcc}
\hline & S2A & S2B \\
\hline $\mathrm{Al}(\%)$ & 3,84 & 3,51 \\
$\mathrm{Ca}(\%)$ & 3,77 & 5,22 \\
$\mathrm{Fe}(\%)$ & 18,4 & 17,5 \\
$\mathrm{Mg}(\%)$ & 2,36 & 3,33 \\
$\mathrm{As}$ & 394 & 306 \\
$\mathrm{Cd}$ & 8,9 & 12,1 \\
$\mathrm{Co}$ & 76,6 & 104 \\
$\mathrm{Cr}$ & 33 & 31 \\
$\mathrm{Cu}$ & 39,8 & 38,9 \\
$\mathrm{Mn}$ & 1603 & 2112 \\
$\mathrm{Ni}$ & 187 & 242 \\
$\mathrm{~Pb}$ & 16,9 & 14,3 \\
$\mathrm{Sr}$ & 98 & 124 \\
$\mathrm{Zn}$ & 2837 & 3769
\end{tabular}

A relação entre as espécies de carbonato dissolvidas e o pH de um ambiente é muito clara. De acordo com Krauskopf, ${ }^{42}$ sob condições naturais, a dissolução da calcita (principal componente do calcário) envolve usualmente ácidos fracos (e.g. Equação 1), mas soluções ácidas fortes devem estar presentes em áreas com ocorrência de intemperismo de pirita (e.g. Equação 2). 
$\mathrm{CaCO}_{3}+\mathrm{H}_{2} \mathrm{CO}_{3} \leftrightarrow \mathrm{Ca}^{2+}+2 \mathrm{HCO}_{3}^{-}$

$\mathrm{CaCO}_{3}+\mathrm{H}^{+} \leftrightarrow \mathrm{Ca}^{2+}+\mathrm{HCO}_{3}^{-}$

Pode ser observado nas Equações 1 e 2 que os íons de $\mathrm{HCO}_{3}^{-}$são originados de diferentes fontes: um tem origem na dissociação do $\mathrm{H}_{2} \mathrm{CO}_{3}$ e o outro é formado pela reação de $\mathrm{H}^{+}$do ácido com $\mathrm{CaCO}_{3}$. Especialmente a Equação 1 se processa em sistema aberto, onde o ácido carbônico está em equilíbrio com $\mathrm{CO}_{2}$ aquoso (dissolvido) que, por sua vez, está em equilíbrio com $\mathrm{CO}_{2}$ atmosférico. ${ }^{43} \mathrm{Na}$ água subterrânea (sistema fechado), a Equação 2 representa o principal tampão de $\mathrm{pH}^{44}$ e pode ser um suprimento adicional de $\mathrm{HCO}_{3}^{-}$para os sistemas fluviais.

$\mathrm{Na}$ área de estudo, o sistema carbonático parece predominar no tamponamento do pH no córrego da Mina. Este sistema de tamponamento, em geral, é complexo e envolve a transferência de carbono entre as fases sólida, líquida e gasosa..$^{43} \mathrm{Em}$ sistema aberto, quando o dióxido de carbono interage com a água, ele dissolverá até que o estado de equilíbrio seja alcançado, com conseqüentes dissociações fortemente controladas pelo $\mathrm{pH}$ :

$$
\begin{array}{ll}
\mathrm{H}_{2} \mathrm{CO}_{3} \leftrightarrow \mathrm{HCO}_{3}^{-}+\mathrm{H}^{+} & \mathrm{K}_{1}=10^{-6,4} \\
\mathrm{HCO}_{3}^{-} \leftrightarrow \mathrm{CO}_{3}{ }^{2-}+\mathrm{H}^{+} & \mathrm{K}_{2}=10^{-10,3}
\end{array}
$$

onde $\mathrm{K}_{1}$ e $\mathrm{K}_{2}$ representam constantes de dissociação. De acordo com a Lei de Ação das Massas, as espécies dominantes na solução serão $\mathrm{H}_{2} \mathrm{CO}_{3}(\mathrm{pH}<6,4), \mathrm{HCO}_{3}^{-}(6,4<\mathrm{pH}<10,3)$ e $\mathrm{CO}_{3}{ }^{2-}(\mathrm{pH}>10,3)$. Embora os sistemas naturais sejam mais complexos do que expressam as Equações 1-4, as condições físico-químicas da água do córrego da Mina corroboram um sistema tampão predito pelas Equações 3 e 4 . O valor de $\mathrm{pH}$ de 6,4 na amostra ANA8 (Tabela 3) corrobora o equilíbrio das atividades de $\mathrm{H}_{2} \mathrm{CO}_{3}{\mathrm{e} \mathrm{HCO}_{3}^{-}}^{-}$e deve ser tamponado de acordo com a Equação 3. O aumento do pH para 10,8 (ANA6) deve ser resultante de incrementos de $\mathrm{HCO}_{3}{ }^{-}$de águas subterrâneas que alimentam o córrego da Mina. Neste caso, o sistema se mostra tamponado possivelmente pela Equação 4, cujo equilíbrio $\left(\mathrm{HCO}_{3}{ }^{-}=\mathrm{CO}_{3}{ }^{2-}\right)$ ocorre em pH de 10,3, acima do qual $\mathrm{HCO}_{3}^{-}<\mathrm{CO}_{3}{ }^{2-}$ (Tabela 3).

\section{A eficiência na remoção de Mn da drenagem ácida}

Um dos problemas que têm sido encontrados em neutralização de DAM por meio de adição de $\mathrm{CaO}$ está relacionado com a deficiência na remoção de $\mathrm{Mn} .{ }^{45} \mathrm{Na}$ Tabela 3 observa-se que a diminuição na concentração de manganês ocorreu apenas quando a corrente ácida atingiu o córrego da Mina, como mostram os valores de ANA8 $\left(2,48 \mathrm{mg} \mathrm{L}^{-1}\right) \mathrm{e}$ ANA6 ( $\left.<0,001 \mathrm{mg} \mathrm{L}^{-1}\right)$ contra $11,8 \mathrm{mg} \mathrm{L}^{-1}$ na DAM original (ANA1). A Figura 3 apresenta os resultados da modelagem geoquímica para as amostras de DAM e do córrego da Mina. As concentrações de Fe(II), $\mathrm{Fe}(\mathrm{III})$ e $\mathrm{Mn}$ (II) estão em $\mathrm{mg} \mathrm{L}^{-1}$ e as respectivas espécies químicas estão em porcentagem. Nota-se que, nas amostras ANA1, ANA2 e ANA8, predominam ferro e manganês na forma iônica $\left(\mathrm{Fe}^{2+} \mathrm{e} \mathrm{Mn}^{2+}\right)$. A diminuição na concentração de $\mathrm{Fe}(\mathrm{II})$ de $256 \mathrm{mg} \mathrm{L}^{-1}$ (ANA2) para $15,8 \mathrm{mg} \mathrm{L}^{-1}$ (ANA8) (Figura 3) contribuiu para a remoção de parte do $\mathrm{Mn}$ (II). Por outro lado, na amostra ANA6 predomina Fe(III) na forma $\mathrm{Fe}(\mathrm{OH})_{4}^{-}$. Os resultados do presente estudo mostraram que em $\mathrm{pH}$ extremamente alcalino (10,8; ponto ANA6 do córrego da Mina) ocorreu condições para a remoção eficaz do manganês, uma vez que acelerou a cinética de oxidação do ferro ferroso para férrico.

\section{Impacto da DAM em drenagem regional}

O impacto físico-químico das águas do córrego da Mina no rio Laranjinha, um dos principais da região, é pouco significativo. $\mathrm{O}$ alto

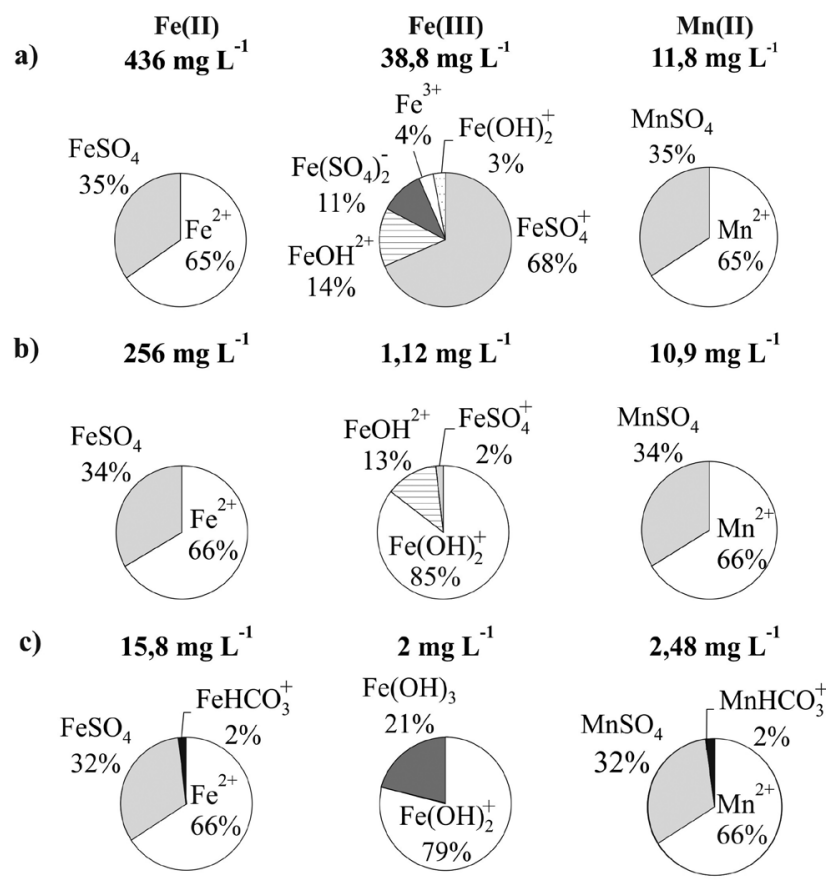

d)

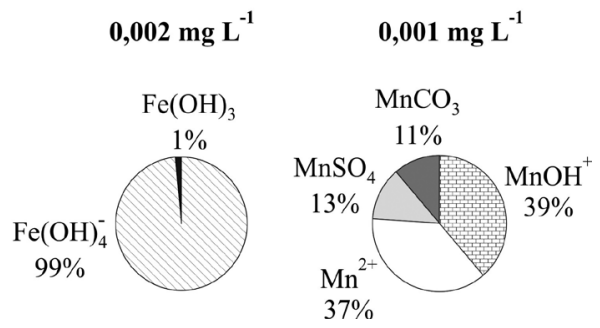

Figura 3. Modelagem geoquímica de Fe e Mn para as amostras: a) ANA1, b) ANA2, c) ANA8 e d) ANA6. Concentrações de $\mathrm{Fe}(\mathrm{II}), \mathrm{Fe}(\mathrm{III})$ e $\mathrm{Mn}(\mathrm{II})$ estão em $\mathrm{mg} \mathrm{L}^{-1}$ e as respectivas espécies químicas estão apresentadas em porcentagem

contraste de vazões destas drenagens (embora não medida neste estudo, a vazão do córrego da Mina é muito inferior ao valor típico de $20 \mathrm{~m}^{3} \mathrm{~s}^{-1}$ no rio Laranjinha ${ }^{46}$ ) deve contribuir para minimizar este impacto. No rio Laranjinha, o pH subiu de 6,2 (ANA5, antes da confluência) para 7,3 (ANA7) após a descarga das águas alcalinas (pH 10,8) do córrego da Mina. O incremento na concentração dos constituintes químicos no rio Laranjinha foi leve em relação ao $\mathrm{Al}, \mathrm{Ca}$ e Fe. Este rio é a principal fonte de abastecimento de água no município de Figueira e, nos pontos de amostragem (a jusante da estação de captação de água), a maioria dos parâmetros físico-químicos analisados (e.g. As, Cd, Mn, $\mathrm{SO}_{4}{ }^{2-}, \mathrm{pH}$ ) ficaram abaixo dos padrões estabelecidos pela Resolução do Conselho Nacional do Meio Ambiente, CONAMA n ${ }^{\circ} 357 / 2005^{47}$ (0,01 mg L ${ }^{-1} \mathrm{As} ; 0,001 \mathrm{mg} \mathrm{L}^{-1} \mathrm{Cd} ; 0,1 \mathrm{mg} \mathrm{L}^{-1} \mathrm{Mn} ; 250 \mathrm{mg} \mathrm{L}^{-1} \mathrm{SO}_{4}{ }^{2-} \mathrm{e}$ $\mathrm{pH}$ no intervalo de 6 a 9). Por outro lado, o nível de $\mathrm{Al}$ nas águas do rio Laranjinha imediatamente após a confluência do córrego da Mina foi levemente superior ao estabelecido pela referida resolução $(0,16$ mg L ${ }^{-1} \mathrm{Al}$ em ANA7, contra $0,1 \mathrm{mg} \mathrm{L}^{-1}$ do CONAMA).

\section{CONCLUSÕES}

Os resultados deste estudo mostraram que as concentrações de As são significativas no carvão das minas de Figueira (PR). Entretanto, os níveis deste metalóide e de outros metais (Cd, Co, Cr, Cu, Ni e $\mathrm{Pb}$ ) foram baixos na drenagem ácida de mina, em contraste com altas concentrações de $\mathrm{Al}, \mathrm{Fe}, \mathrm{Mn}, \mathrm{SO}_{4}{ }^{2-}$ e $\mathrm{Zn}$. Os processos físicoquímicos atuantes na neutralização da drenagem ácida com $\mathrm{CaO}$ são 
complexos, parecendo que o $\mathrm{pH}$ de hidrólise de cada constituinte químico é um fator determinante em sua precipitação. $\mathrm{O}$ sistema de tamponamento carbonático natural na área investigada, devido à presença de rochas calcárias, forneceu condições físico-químicas mais alcalinas necessárias para a retirada de elementos dissolvidos, residuais do processo de neutralização com $\mathrm{CaO}$ (exceto $\mathrm{Al}$ ). Modelagem geoquímica mostrou que a remoção de Mn bivalente dissolvido ocorreu com eficiência apenas após a precipitação do ferro bivalente, em condições alcalinas extremas. Nestas condições, Al foi solubilizado de minerais silicáticos e ocorreu incremento no seu nível de concentração nas águas fluviais.

\section{AGRADECIMENTOS}

Ao CNPq e à CAPES pelo apoio financeiro; à Carbonífera do Cambuí Ltda. pelo apoio logístico durante a amostragem de campo; ao Instituto de Geociências da UNICAMP pela utilização de laboratórios; aos Profs. B. R. Figueiredo, J. Enzweiler e a dois revisores anônimos pelas sugestões e críticas no manuscrito original.

\section{REFERÊNCIAS}

1. Akcil, A.; Koldas, S.; Journal of Cleaner Production 2006, 14, 1139.

2. Blodau, C.; Sci. Total Environ. 2006, 369, 307.

3. Cravotta III, C. A.; Trahan, M. K.; Appl. Geochem. 1999, 14, 581.

4. Kalin, M.; Fyson, A.; Wheeler, W. N.; Sci. Total Environ. 2006, 366, 395.

5. Maree, J. P.; du Plessis, P.; van der Walt, C. J.; Water Sci. Technol. 1992, 26,345 .

6. Hammarstrom, J. M.; Sibrell, P. L.; Belkin, H. E.; Appl. Geochem. 2003, 18,1705 .

7. Santomartino, S.; Webb, J. A.; Appl. Geochem. 2007, 22, 2344.

8. Potgieter-Vermaak, S. S.; Potgieter, J. H.; Monama, P.; Van Grieken, R.; Miner. Eng. 2006, 19, 454.

9. Maree, J .P.; du Plessis, P.; Water Sci. Technol. 1994, 29, 285.

10. Sikora, F. J.; Behrends, L. L.; Brodie, G. A.; Taylor, H. N.; Water Environ. Res. 2000, 72, 536.

11. Hallberg, K. B.; Johnson, D. B.; Sci. Total Environ. 2005, 338, 115.

12. Gazea, B.; Adam, K.; Kontopolus, A.; Miner. Eng. 1996, 9, 23.

13. Pampêo, M. L. M.; Moschini, V. C.; Alexandre, N. Z.; Santo, E.; Acta Scientiarum, Biological Sciences 2004, 26, 125.

14. Soares, E. R.; Mello, J. W. V.; Schaefer, C. E. G. R.; Costa, L. M.; R. Bras. Ci. Solo 2006, 30, 171.

15. Flues, M.; Hama, P.; Fornaro, A.; Quim. Nova 2003, 26, 479.

16. Fungaro, D. A.; Izidoro, J. C.; Quim. Nova 2006, 29, 735.

17. Shuqair, S. M. S.; Tese de Doutorado, Universidade de São Paulo, Brasil, 2002.

18. Schneider, R. L.; Mühlmann, H.; Tommasi, E.; Medeiros, R. A.; Daemon, R. F.; Nogueira, A. A.; Anais do XXVIII Congresso Brasileiro de Geologia, Porto Alegre, Brasil, 1974.

19. Campaner, V. P.; Spoladore, A. Em Estudos do Complexo Carboelétrico de Figueira; Fungaro, D. A.. org.; Cubo Multimídia: São Paulo, 2007, cap. 1, CD-ROM (ISBN: 978-85-60064-03-8).

20. Jardim, W. F.; Fadini, P. S.; The Analyst 2000, 125, 549.

21. Nordstrom, D. K.; Wilde, F. D. Em National Field Manual for the
Collection of Water-Quality Data; Wilde, F. D.; Radtke, B. D.; Gibs, J.; Iwatsubo, R. T., eds.; U. S. Geological Survey Techniques in WaterResources Investigations: Reston, 1998, book 9, cap. A6.

22. Concas, A.; Ardau, C.; Cristini, A.; Zuddas, P.; Cao, G.; Chemosphere 2006, 63, 244.

23. Winston, R. B.; Graphical User Interface for MODFLOW; Geological Survey Open-File Report 00-315, 2000.

24. Parkhurst, D. L.; Appelo, C. A. J.; User's guide to PHREEQC: Computer Program for Speciation, Reaction-path, 1D-transport, and Inverse Geochemical Calculations; U. S. Geological Survey Water-Resources Investigations Report, 1999.

25. Stumm, W.; Morgan, J. J.; Aquatc chemistry, $2^{\text {nd }}$ ed., John Willey: New York, 1981.

26. Merkel, B. J.; Friedrich, P. B.; Grundwasserchemie: Praxisorientier Leitfaden zur numerischen Modellierung von Beschaffenheit, Kontamination und Sanierung aquatischer Systeme, Springer: Berlin, 2002.

27. Taylor, S. R.; McLennan, S. M.; The continental crust: its composition and evolution, Oxford: England, 1985.

28. Christanis, K.; Georgakopoulos, A.; Fernández-Turiel, J. L.; Bouzinos, A.; Int. J. Coal Geol. 1998, 36, 313.

29. Song, D.; Qin, Y.; Zhang, J.; Wang, W.; Zheng, C.; Int. J. Coal Geol. 2007, 69, 179.

30. Gayer, R. A.; Rose, M.; Dehmer, J.; Shao, L. Y.; Int. J. Coal Geol. 1999 , $40,151$.

31. van der Flier-Killer, E.; Goodarzi, F.; Bull. Geol. Soc. France 1991, 162, 255.

32. Dai, S.; Zeng, R.; Sun, Y.; Int. J. Coal Geol. 2006, 66, 217.

33. Pereira, W. C.; Tese PhD, Universidade de Barcelona, Espanha, 1996.

34. Kalkreuth, W.; Holz, M.; Kern, M.; Machado, G.; Mexias, A.; Silva, M. B.; Willett, J.; Finkelman, R.; Burger, H.; Int. J. Coal Geol. 2006, 68, 79.

35. Chon, H.-T.; Hwang, J.-H.; Environ. Geochem. Health 2000, 22, 155.

36. Licht, O. A. B.; Prospecção Geoquímica: Princípios, Técnicas e Métodos, CPRM: Rio de Janeiro, 1998.

37. Bednar, A. J.; Garbarino, J. R.; Ranville, J. F.; Wildeman, T. R.; J. Geochem. Explor. 2005, 85, 55.

38. Casiot, C.; Lebrun, S.; Morin, G.; Bruneel, O.; Personné, J. C.; ElbazPoulichet, F.; Sci. Total Environ. 2005, 147, 122.

39. Andrade, R. P.; Dissertação de Mestrado, Universidade Estadual de Campinas, Brasil, 2007.

40. Levinson, A. A.; Introduction to Exploration Geochemistry, Illinois: Applied Publishing, 1974.

41. Gzyl, G.; Banks, D.; J. Contam. Hydrol. 2007, 92, 66.

42. Krauskopf, K. B.; Introduction to Geochemistry, McGraw-Hill: New York, 1967.

43. Sherlock, E. J.; Lawrence, R. W.; Poulin, R.; Environ. Geol. 1995, 25 , 43.

44. Morin, K. A.; Cherry, J. A.; J. Contam. Hydrol. 1988, 2, 323.

45. Sibrell, P. L.; Watten, B.; Boone T.; Hydrometallurgy: Fifth International Conference in Honor of Professor Ian Ritchie, Electrometallurgy and Environmental Hydrometallurgy, 2003.

46. SUDERHSA; Qualidade das águas interiores do Estado do Paraná (1987-1995), SUDERHSA: Curitiba, 1997.

47. http://www.mma.gov.br, acessada em Agosto 2007. 